\title{
Picture-naming norms for Canadian French: Name agreement, familiarity, visual complexity, and age of acquisition
}

\author{
MÉLANIE SIROIS \\ Université du Québec, Montréal, Canada \\ HELGARD KREMIN \\ Centre National de la Recherche Scientifique, Paris, France \\ and \\ HENRI COHEN \\ Université du Québec, Montréal, Canada
}

\begin{abstract}
The present study provides Canadian French normative data for 388 line drawings from the European Picture Pool for Oral Naming (Protocole européen de dénomination orale d'images; PEDOI; Kremin et al., 2003). One hundred eighty subjects were equally distributed for age group (18-39, 40-59, 60-85), educational level (low, high), and sex. They rated pictures of objects on age of acquisition, name agreement, familiarity, and visual complexity. Syllable length and word frequency were also taken into account. The present study suggests that age of acquisition and name agreement show significant age-related differences. These results show that unequivocal interpretation of age-related differences can be made when age-appropriate norms are used.
\end{abstract}

The picture-naming task has been widely used to investigate spoken word production. It requires subjects to quickly say the name of an object after looking at its pictorial representation. Subjects visually analyze the picture to extract features that are then compared with stored information in order to choose an appropriate word name. One way of investigating the processes underlying picture naming is by varying individual item characteristics, such as word frequency, age of acquisition, or name agreement, and observing how they affect picture-naming performance. It is important to control for all essential variables that might have an effect on naming as well as those that are strongly correlated. If not, one's conclusions are likely to be erroneous. Some authors have reported frequency effects (Forster \& Chambers, 1973; Jescheniak \& Levelt, 1994; Oldfield \& Wingfield, 1965), but did not control for strongly correlated variables such as age of acquisition (AoA). Their results have now been called into question (e.g., Barry, Hirsch, Johnston, \& Williams, 2001; Chalard,

This research was supported, in part, by scholarships from the National Sciences and Engineering Council of Canada, the Fonds québécois de la recherche sur la nature et les technologies, and grants from the Social Sciences and Humanities Research Council. We thank Jenny Staab, Sara Bélanger, and Valérie Bédirian for helping with data collection and François Labelle for drawing the pictures. We also thank Patrick Bonin and an anonymous reviewer for their comments on an earlier version of this article. Correspondence concerning this article should be addressed to H. Cohen, CNC, UQAM, C.P. 8888, succ. Centre-Ville, Montréal, PQ, H3C 3P8 Canada (e-mail: cohen.henri@uqam.ca).
Bonin, Méot, Boyer, \& Fayol, 2003). Standardized picture pools have been used extensively in an attempt to tease out the effects of these variables on naming reaction times (Bates, Burani, D’Amico, \& Barca, 2001; Bonin, Peereman, Malardier, Méot, \& Chalard, 2003; Cuetos, Ellis, \& Alvarez, 1999; Kremin, Hamerel, Dordain, De Wilde, \& Perrier, 2000; Snodgrass \& Yuditsky, 1996).

The norms collected across these normative studies concerned AoA as well as many other variables, such as name agreement, word frequency, familiarity, visual complexity, and word length. Name agreement is usually defined as the proportion of subjects who give the same name to an object. Many studies have shown that pictures of objects with a single dominant response are named more rapidly and more accurately than those with multiple responses. This effect is robust and appears to be independent of other attributes (Barry, Morrison, \& Ellis, 1997; Gilhooly \& Gilhooly, 1979; Lachman, Shaffer, \& Hennrikus, 1974; Paivio, Clark, Digdon, \& Bons, 1989; Vitkovitch \& Tyrrell, 1995).

There is a significant relationship between object familiarity and other variables, such as age of acquisition and word frequency. When the variance attributed to this relationship is removed, object familiarity has been found to contribute little unique variance to the prediction of item differences in picture-naming latency (Barry et al., 1997; Cuetos et al., 1999; Snodgrass \& Yuditsky, 1996). Visual complexity refers to the amount of detail in a picture and can affect the time it takes to recognize a picture as the representation of a familiar object (Ellis \& Mor- 
rison, 1998). Its effect on naming response time (RT) has been reported in some studies (Ellis \& Morrison, 1998; Vitkovitch \& Tyrrell, 1995), but not in others (Bonin, Chalard, Méot, \& Fayol, 2002; Cuetos et al., 1999; Snodgrass $\&$ Yuditsky, 1996). Thus, its effect on picture naming does not seem robust. Similarly, word length, or the number of phonemes or syllables in a word, was not shown to be a reliable contributor to picture-naming time. Although certain studies have found an independent word length effect (Cuetos et al., 1999; Morrison, Ellis, \& Quinlan, 1992; Paivio et al., 1989), several other studies did not (Bachoud-Lévi, Dupoux, Cohen, \& Mehler, 1998; Carroll \& White, 1973; Snodgrass \& Yuditsky, 1996).

Until recently, the frequency with which a word occurs in the language was perceived to be a robust determinant of the latency and success with which a picture can be named. The usual explanation for these results was that the representations of words would become accessible more rapidly as a result of repeated activation (Balota \& Chumbley, 1990; Forster \& Chambers, 1973; Huttenlocher \& Kubicek, 1983; Oldfield \& Wingfield, 1965; see Monsell, 1991, for a review). However, there is now growing agreement that at least some of these frequency effects are in fact AoA effects. Age of acquisition refers to the age at which a word was first learned. It seems that words learned earlier in life tend to be named faster than words learned later in life. When the correlation between word frequency and AoA is taken into account, some studies have found strong effects of AoA but no effect of word frequency on naming RT (Barry et al., 2001; Bonin et al., 2002; Dell'Acqua, Lotto, \& Job, 2000; Kremin et al., 2001; Morrison et al., 1992). On the other hand, others have reported significant and independent effects of both variables (Barry et al., 1997; Bates et al., 2001; Bonin et al., 2003; Cuetos et al., 1999; Ellis \& Morrison, 1998; Meschyan \& Hernandez, 2002).

However, these results relied mostly on adult ratings of word-learning age (rated AoA), and it was unclear whether or not rated AoA is a valid indicator of the measure it claims to represent. To address this question, some authors derived a more objective measure by evaluating the vocabulary of a group of children (Chalard et al., 2003; Gilhooly \& Gilhooly, 1980; Morrison, Chappell, \& Ellis, 1997). These studies suggest there is a good relationship between rated AoA and objective AoA, demonstrating that subjective measures are valid and reliable alternatives (Gilhooly \& Gilhooly, 1980; Morrison et al., 1997).

It is only recently that normative data have been collected in several different linguistic and sociocultural contexts. It was found that name agreement yields rather weak cross-linguistic correlations (between .20 and .50) and seems to depend more on language and culture than any other variable (Dell'Acqua et al., 2000; Kremin et al., 2003). Moreover, differences were also observed for object familiarity, suggesting that objects that are common in a given culture might be judged differently in another (Cuetos et al., 1999). There is also evidence that dominant names change, over generations, and that AoA values that are appropriate for younger generations are not appropriate for older ones (Hodgson \& Ellis, 1998).

It thus appears that several factors may affect how object attributes are judged, perceived, or named. The effect of such variables must therefore be taken into account when investigating how normal or brain-damaged individuals rate or name objects and their attributes. Finally, normative data collected for older subjects is lacking. Data are more often collected from young, educated subject samples.

From this perspective, the present study aims to provide researchers with a normative database for pictorial material that will be useful with subjects whose first language is Canadian French. In this study, we control for individual factors as well as name agreement, object familiarity, visual complexity, and AoA.

\section{METHOD}

\section{Subjects}

A total of 180 different healthy volunteers were recruited. They were all native speakers of Canadian French. The subjects were equally distributed for age group (18-39, 40-59, 60-85), educational level (low, high), and sex. High and low levels of education were based on Statistics Canada (1996) median schooling for each age group. In the oldest age group, the subjects were considered highly educated if they had completed 10 years of schooling. In the other two groups, the subjects were considered highly educated if they had completed 11 years of schooling. The subjects were recruited through ads placed in newspapers or on community billboards, as well as through various community recreation centers. Individuals were excluded if they reported a medical condition known to affect cognition or vision (e.g., brain surgery, epilepsy, Parkinson's disease, or cataracts) or a recent history (in the past three years) of significant mental health problems such as depression or substance abuse. The elderly subjects were not screened for dementia.

\section{Materials}

The stimuli were a set of 388 black-and-white line drawings adapted from the European Standardized Picture Pool for Oral Naming (Protocole européen de dénomination orale d'images; PEDOI), a new multilanguage picture pool standardized in eight European languages as well as in Latin American Spanish (Kremin et al., 2003). It includes exemplars from several categories, and all the objects are depicted at the basic level of categorization. All the pictures were redrawn by a graphics artist using Adobe Illustrator. About 15 original drawings that did not resemble the objects in use in Canada (e.g., farm) and those that lacked clarity were revised.

\section{Procedure}

Five different booklets containing the 388 pictures in a random order were prepared, with each page containing six pictures ranging in size from approximately $5 \times 5 \mathrm{~cm}$ to $10 \times 10 \mathrm{~cm}$. Each of the five versions of the booklet was given to only 1 subject in each sociodemographic group (e.g., all young educated men received a different version). For each picture, the subjects were asked to write their answers on separate answer sheets. A practice session comprising six trials was conducted before each experimental task. The subjects were tested in groups of 6 or less. Halfway through the task, the subjects were given a 5 -min rest period.

Name agreement and familiarity. The subjects were first asked to write down the usual name they would give to the object depicted in each picture and to be as precise as possible. If no name was available, they had to write "do not know." They were then asked to 
judge the familiarity of the object depicted, according to the degree to which they had been in contact with or thought about it. They were instructed to rate familiarity on a 5-point scale, where $1=$ not at all familiar and $5=$ very familiar. The procedure and the rating instructions were taken from Barry et al. (1997). Following Snodgrass and Vanderwart (1980), a strict criterion was used for counting different instances of names when computing name agreement values. When the name given by a subject was similar but not identical to an established name, it was computed as different.

Visual complexity. The subjects were also asked to judge how complex the picture of an object was in terms of its details. They were instructed to rate the complexity of the picture itself, rather than what the object represented. A 5-point scale was used, where $1=$ not at all complex and $5=$ very complex. Rating instructions closely followed those of Snodgrass and Vanderwart (1980).

Age of acquisition. A different procedure was adopted in this task: The most common name (following the name agreement task) was presented instead of the depicted object, and the subjects wrote their answers directly in their booklets; each page contained 25 modal names. The subjects were asked to estimate the age at which they believed they had learned each of these names. Raters were asked to circle the appropriate number for each word on a scale ranging from 1 (learned before age 2) to 7 (learned at age 13 or over). The rating scale was presented at the top of each page, as a reminder. Rating instructions were the same as those used in Gilhooly and Logie (1980).

\section{RESULTS AND DISCUSSION}

\section{Outlying Subjects and Problematic Objects}

The summary statistics, as well as all the later analyses, were calculated after the data of 3 subjects and 38 problematic items were removed, following the procedure described below. In each task, for each item, scores greater than three $S D$ s were identified. The data of 2 subjects for the visual complexity task and of 1 subject for the familiarity task were excluded from the analysis because a high percentage of their ratings $(3.6 \%-4.9 \%)$ qualified as outliers with respect to the group average $(0.3 \%-0.55 \%$ of the ratings were outliers). These subjects may have misunderstood the rating instructions or they may have skipped items, resulting in assigning ratings unrelated to the appropriate picture.

Overall, 38 pictures of objects were removed from the analyses. They are presented in the Appendix. Twenty-six items were removed because the subjects failed to reach
Table 1

Mean Age, Mean Education in Years, and Standard Deviations for Each Task and Each Age Group

\begin{tabular}{|c|c|c|c|c|c|c|}
\hline \multirow[b]{2}{*}{ Group } & \multirow[b]{2}{*}{ Task } & \multirow[b]{2}{*}{$N$} & \multicolumn{2}{|c|}{ Age } & \multicolumn{2}{|c|}{$\begin{array}{c}\text { Education } \\
\text { (years) }\end{array}$} \\
\hline & & & $M$ & $S D$ & $M$ & $S D$ \\
\hline \multirow{3}{*}{ Young } & AoA & 20 & 25.75 & 4.93 & 12.70 & 2.41 \\
\hline & VC & 19 & 25.44 & 5.83 & 12.89 & 2.09 \\
\hline & NA \& Fam & 20 & 26.65 & 4.85 & 12.80 & 3.37 \\
\hline \multirow[t]{3}{*}{ Middle aged } & AoA & 20 & 50.60 & 4.87 & 12.55 & 3.50 \\
\hline & $\mathrm{VC}$ & 20 & 49.50 & 5.29 & 12.30 & 2.52 \\
\hline & NA \& Fam & 19 & 48.89 & 3.22 & 12.56 & 3.13 \\
\hline \multirow[t]{3}{*}{ Older } & AoA & 20 & 66.05 & 4.42 & 10.05 & 4.06 \\
\hline & VC & 19 & 67.44 & 5.39 & 10.56 & 3.78 \\
\hline & NA \& Fam & 20 & 69.70 & 4.88 & 10.30 & 3.11 \\
\hline
\end{tabular}

Note-AoA, age of acquisition; VC, visual complexity; NA \& Fam, name agreement and familiarity task.

a name agreement criterion of $33 \%$. These items may have been unclear or may have represented objects not commonly used in Canada. Twelve objects were removed because their names were polysemous (e.g., in French, "trombone" refers to a paper clip as well as to a musical instrument) and therefore it was impossible for the subjects to give an accurate estimate of the AoA based only on the name of the object.

\section{Normative Data Description}

The summary of the rating data obtained from our sample of Canadian French-speaking subjects is available at www.crsc.uqam.ca/Appendices_SiroisNormsCndFr.pdf. The percentage of subjects producing the most common name, mean AoA, familiarity, and visual complexity are presented for each item, and data are divided per age group. Word frequency, syllable length, and the most frequent alternate names are also given. Items that show no sociodemographic difference and items that show at least one sociodemographic difference are available in different Internet files.

Table 1 presents a summary of the sociodemographic characteristics of the three different age groups and Table 2 shows the summary statistics for the following measures: the percentage of subjects producing the modal name, AoA, object familiarity, visual complexity, and two

Table 2

Summary Statistics for All Variables

\begin{tabular}{lccccrrr}
\hline & NA & AoA & Fam & VC & Freq1 & Freq2 & Length \\
\hline Mean & 0.75 & 3.40 & 3.26 & 2.95 & 120.34 & 100.88 & 2.16 \\
SD & 0.20 & 1.04 & 0.82 & 0.86 & 59.80 & 57.67 & 0.93 \\
Median & 0.78 & 3.27 & 3.25 & 2.92 & 111.39 & 94.65 & 2.00 \\
Min & 0.35 & 1.44 & 1.59 & 1.00 & 30.10 & 1.28 & 1.00 \\
Max & 1.00 & 6.09 & 4.88 & 4.75 & 281.09 & 267.94 & 6.00 \\
Q1 & 0.58 & 2.68 & 2.60 & 2.25 & 71.88 & 55.02 & 2.00 \\
Q3 & 0.93 & 4.19 & 3.95 & 3.67 & 164.10 & 139.39 & 3.00 \\
Range & 0.65 & 4.64 & 3.29 & 3.75 & 250.99 & 266.66 & 5.00 \\
$N$ & 350 & 350 & 350 & 350 & 288 & 307 & 350 \\
\hline
\end{tabular}

Note-NA, name agreement; AoA, age of acquisition; Fam, familiarity task; VC, visual complexity; Freq1, frequency (taken from Baudot, 1992); Freq2, frequency (taken from New et al., 2001); Length, in syllables; Q1, 25th percentile; Q3, 75th percentile. 
Table 3

Correlation Matrix

\begin{tabular}{lrrrrrr}
\hline & \multicolumn{1}{c}{ NA } & \multicolumn{1}{c}{ AoA } & Fam & VC & Freq1 & Freq2 \\
\hline AoA & -0.318 & & & & & \\
Fam & n.s. & -0.416 & & & & \\
VC & n.s. & 0.328 & -0.376 & & & \\
Freq1 & 0.156 & -0.427 & 0.280 & n.s. & & \\
Freq2 & 0.229 & -0.517 & 0.396 & n.s. & 0.845 & \\
Length & -0.172 & 0.422 & n.s. & 0.196 & -0.201 & -0.356 \\
\hline
\end{tabular}

Note-NA, name agreement; AoA, age of acquisition; Fam, familiarity task; VC, visual complexity; Freq1, frequency (taken from Baudot, 1992); Freq2, frequency (taken from New et al., 2001); Length, in syllables.

frequency measures. Frequency values (Freq1 \& Freq2) were taken, respectively, from Baudot (1992), which is the most up-to-date frequency database for Canadian French, and from New, Pallier, Ferrand, and Matos (2001), which represents the largest contemporary French database. Following the procedure used by Barry et al. (1997), all frequency counts were transformed using the formula $\log (1+\mathrm{x}) * 100$, to reduce skewness.

\section{Correlations Between the Measures}

Table 3 shows the matrix of significant correlations for all the variables. Because frequency measures were available for only 288 (Freq1) and 307 (Freq2) of the 388 items, a separate set of correlations was computed for these subsets. As expected, the two measures of frequency show a high positive correlation (.85). Correlations between name agreement and other variables are low, except for AoA ( -.33$)$. This result suggests that there is less agreement on the names of objects that are learned later in life. Although the name agreement task and the familiarity task were completed simultaneously, there is no correlation at all (.03) between these tasks, so contamination is unlikely. Correlations between AoA and other measures are high (between .33 and .52), suggesting that objects we learn about earliest in life are the simplest and most familiar objects. They also have shorter names and are encountered more frequently in Canadian French. Finally, as expected, word frequency correlated significantly with object familiarity.

\section{Comparison With Other French Normative Data}

To verify the impact of cultural variables, we compared our data with normative data obtained in France. We computed means and correlational analyses for 155 items common to Alario and Ferrand's (1999) database, the French PEDOI (Kremin et al., 2003), and our database. Bonin et al.'s (2003) database was excluded from the analyses, having only 41 common items.

As shown in Table 4, Kremin et al. (2003) and Alario and Ferrand (1999) obtained similar familiarity and name agreement mean scores, but Kremin et al.'s visual complexity scores were slightly lower. However, our results were different. The name agreement (NA) and visual complexity (VC) mean scores are significantly lower [NA, $F(2,154)=$ 16.01 , and $\mathrm{VC}, F(2,154)=16.91]$ and the object familiarity mean score is significantly higher $[F(2,154)=5,76]$. Overall, these results suggest that French Canadian subjects rate items differently than do French subjects.

As shown in Table 5, the correlational analysis between the three databases suggests that there is a strong relationship between AoA values, familiarity values, and to a lesser extent, visual complexity values. Although they are significant, the relationships between name agreement values are much weaker. The stronger correlations obtained for AoA and familiarity can also be explained, in part, by the fact that these ratings are less dependent on the pictorial stimuli, when compared with name agreement and visual complexity judgments. These results also suggest that culture has a significant impact on name agreement. From one region or country to another, people use different words to name the same object. This result is consistent with other comparative studies (Dell'Acqua et al., 2000; Kremin et al., 2003; Sanfeliu \& Fernandez, 1996).

Except for visual complexity, all correlations between Alario and Ferrand's database and Kremin's database are stronger than the correlations between Alario and Ferrand's database and ours. Taken together, these results also suggest that culture has a differential impact on each item.

Table 4

Means and Standard Deviations for All Variables: French and Canadian French Samples

\begin{tabular}{|c|c|c|c|c|c|c|c|c|}
\hline & \multicolumn{2}{|c|}{ NA } & \multicolumn{2}{|c|}{ AoA } & \multicolumn{2}{|c|}{ Fam } & \multicolumn{2}{|c|}{$\mathrm{VC}$} \\
\hline & $M$ & $S D$ & $M$ & $S D$ & $M$ & $S D$ & $M$ & $S D$ \\
\hline Sirois et al. & 0.84 & 0.16 & 3.04 & 0.97 & 3.31 & 0.87 & 2.69 & 0.78 \\
\hline Kremin et al. & 0.91 & 0.10 & 2.71 & 0.94 & 2.97 & 1.22 & 3.32 & 1.10 \\
\hline Alario and Ferrand & 0.92 & 0.14 & $-^{*}$ & - & 2.91 & 1.23 & 3.04 & 0.96 \\
\hline
\end{tabular}

Note-NA, name agreement; AoA, age of acquisition; Fam, familiarity task; VC, visual complexity. *Alario and Ferrand's AoA rating scores are not presented here because a different rating scale was used in their study. 
Table 5

Significant Correlations Among the Measured Variables in the French and Canadian French Samples

\begin{tabular}{lcccc}
\hline & NA & AoA & Fam & VC \\
\hline Sirois vs. A\&F & .376 & .829 & .898 & .758 \\
Sirois vs. Kremin & .527 & .836 & .928 & .783 \\
Kremin vs. A\&F & .485 & .867 & .953 & .700 \\
\hline
\end{tabular}

Note-NA, name agreement; AoA, age of acquisition; Fam, familiarity task; VC, visual complexity; A\&F, results from Alario \& Ferrand (1999); Kremin, results from Kremin et al. (2003).

However, the overall correlational structure stays similar from one cultural group to another.

\section{Sociodemographic Variables}

For each measure, the influence of sociodemographic variables (age, education, and sex) was verified. Table 6 presents the same summary statistics as Table 2, but for each age group. We also performed correlational analyses on the data obtained for each age group. There was no difference between the correlation coefficients $(p>.10)$. Separate ANOVAs and two-tailed $t$ tests were conducted to compare the mean ratings given by each sociodemographic group for each measure. These results are described in more detail below. Moreover, for each object, mean ratings of object familiarity, visual complexity, and AoA were also computed in order to identify the objects whose ratings differed significantly according to the age, sex, or level of education of the subjects. ANOVAs or two-tailed $t$ tests were also computed on each item. For the name agreement measure, we used a slightly different procedure. Scores that differed more than 35\% were categorized as different. This method was preferred to chi-square analysis, because the frequencies were not of the expected size in more than $40 \%$ of the cases. Overall, 177 items showed no significant difference (age, sex, or education level), although 173 (almost 50\%) did show one or more significant differences. The total number of significant differences is presented in Table 7 , by variable and by sociodemographic group.

AoA. Mean AoA ratings differed significantly according to age $[F(2,1047)=23.31]$ and level of education $[t(698)=3.51]$. The younger subjects estimated that they learned words earlier than the middle-aged and older subjects. Subjects with a higher level of education also estimated that they learned words earlier than subjects with a lower level of education. Sex had no significant influence on ratings $[t(698)=0.59, p=.55]$. Per-item results were consistent with the above overall measure results. For almost $25 \%$ of objects, AoA ratings differed significantly according to age. To a lesser extent, ratings also differed according to level of education (see Table 5).

Name agreement. Significant age-related differences were found $[F(2,1047)=6.24]$, suggesting that younger subjects agree more on the name given to an object than older subjects. Level of education and sex had no significant influence on name agreement $[t(698)=1.83, p=$ $.07 ; t(698)=1.55, p=.12]$. The per-item analyses indicated that almost $12 \%$ of objects differ significantly according to age.

Familiarity. There was a significant age-related difference $[F(2,1047)=20.32]$. The older subjects tended to rate objects as more familiar than did the two other groups. There was no education-related difference $[t(698)=2.22$, $p=.03]$ or sex-related difference $[t(698)=1.15, p=$ .25]. Interestingly, sex-related differences, although small, were apparent in the per-item analysis (16 objects differed significantly). The per-item analyses also showed an agerelated difference (18 items).

Visual complexity. There were no significant differences related to age $[F(2,1047)=2.80, p=.06]$, level of education $[t(698)=3.51, p=.91]$, or $\operatorname{sex}[t(698)=$ 2.01, $p=.04]$.

These results suggest that the younger the subjects are, the higher the name agreement score is. The difference between the two groups was that the older subjects answered "don't know" more often $(2.78 \%)$ than did the middleaged $(1.55 \%)$ subjects. Because the education cutoff was based on the last census and was different from one group to another, this difference could simply be due to the lower education level of the older subjects. As suggested by Hodgson and Ellis (1998), it is also possible that when an object has more than one plausible name, older subjects have more difficulties accessing it because the activation reaching the lexicon from semantics is divided between these alternatives. It would also have been interesting to

Table 6 Summary Statistics for Young, Middle-Aged, and Older Adults

\begin{tabular}{|c|c|c|c|c|c|c|c|c|c|c|c|c|}
\hline & \multicolumn{3}{|c|}{ NA } & \multicolumn{3}{|c|}{ AoA } & \multicolumn{3}{|c|}{ Fam } & \multicolumn{3}{|c|}{$\mathrm{VC}$} \\
\hline & $\mathrm{Y}$ & M & $\mathrm{O}$ & $\mathrm{Y}$ & M & $\mathrm{O}$ & Y & M & $\mathrm{O}$ & $\mathrm{Y}$ & M & $\mathrm{O}$ \\
\hline Mean & 0.78 & 0.74 & 0.72 & 3.08 & 3.38 & 3.66 & 3.12 & 3.18 & 3.50 & 3.07 & 2.98 & 2.92 \\
\hline Median & 0.85 & 0.80 & 0.75 & 2.98 & 3.25 & 3.45 & 3.00 & 3.16 & 3.55 & 3.05 & 2.90 & 2.92 \\
\hline$S D$ & 0.21 & 0.21 & 0.22 & 0.95 & 1.14 & 1.26 & 0.91 & 0.82 & 0.81 & 0.83 & 0.89 & 0.81 \\
\hline Range & 0.95 & 0.90 & 0.85 & 4.40 & 5.10 & 5.17 & 3.50 & 3.42 & 3.53 & 3.68 & 3.60 & 3.84 \\
\hline Min & 0.05 & 0.10 & 0.15 & 1.30 & 1.25 & 1.41 & 1.45 & 1.42 & 1.42 & 1.05 & 1.15 & 1.00 \\
\hline $\operatorname{Max}$ & 1.00 & 1.00 & 1.00 & 5.70 & 6.35 & 6.58 & 4.95 & 4.84 & 4.95 & 4.74 & 4.75 & 4.84 \\
\hline Q1 & 0.65 & 0.60 & 0.55 & 2.35 & 2.50 & 2.66 & 2.35 & 2.53 & 2.89 & 2.47 & 2.25 & 2.33 \\
\hline Q3 & 0.95 & 0.90 & 0.90 & 3.70 & 4.20 & 4.68 & 3.91 & 3.84 & 4.18 & 3.75 & 3.70 & 3.53 \\
\hline$N$ & 350 & 350 & 350 & 350 & 350 & 350 & 350 & 350 & 350 & 350 & 350 & 350 \\
\hline
\end{tabular}

Note-NA, name agreement; AoA, age of acquisition; Fam, familiarity task; VC, visual complexity; Y, young; $\mathrm{M}$, middle aged; O, older; Q1, 25th percentile; Q3, 75th percentile. 
Table 7

Number of Objects for Which Ratings Differed Significantly According to Age, Level of Education, or Sex

\begin{tabular}{lrrrr}
\hline & NA & AoA & VC & Fam \\
\hline Age & 41 & 86 & 14 & 18 \\
Education & 3 & 27 & 1 & 2 \\
Sex & 6 & 4 & 6 & 16 \\
\hline
\end{tabular}

Note-NA, name agreement; AoA, age of acquisition; VC, visual complexity; Fam, familiarity task.

differentiate between tip-of-the-tongue and "don't know" responses, since the tip-of-the-tongue phenomenon is more frequent in older people (Brown, 1991).

It is more difficult to explain why the youngest group has a higher name agreement score than the two other groups. "Don't know" answers are as frequent as in the middle-aged group. The younger subjects might use fewer synonyms to name the objects. A sociolinguistic study supports this hypothesis by suggesting that older and less educated people use more Canadianisms than do younger people (Paquot, 1988). However, even if we include frequent alternative words (at least two instances in one given age group) in the total name agreement score, there is still a significant difference between the youngest group and the two other groups. This suggests that a greater number of middle-aged and older subjects wrote unique responses. This cohort effect could possibly be explained by various cultural and educational setting differences.

The results also suggest that older and middle-aged subjects did not learn all words at the same age as younger subjects. It is possible that these age-related differences are actually differences in estimation rather than differences in AoA. To verify this hypothesis, the means and standard deviation scores were recalculated after removing all the significantly different AoA rating scores (86 items). There is no longer a significant difference between the middle-aged group $(M=3.25, S D=1.11)$ and the older group $(M=3.35, S D=1.10)$, although the difference between these groups and the youngest group $(M=$ $3.07, S D=0.99$ ) remains. We also looked at the relationships between the AoA rating scores per age group. The correlations between the age groups are strong (from .89 to .95) and suggest that most words were learned in the same order. However, when the 86 different items are included in the analyses, the correlation between the youngest and oldest group AoA rating scores is significantly lower (.76). Taken together, these results suggest that at least part of the difference between the groups can be explained by an actual difference in AoA and not a difference in estimation.

\section{CONCLUSION}

The main goal of the present research was to collect normative data for pictorial stimuli that could be used by researchers interested in memory, language, and other cognitive processes, especially those who work with $\mathrm{Ca}-$ nadian French-speaking samples. This new database also adds to the European Standardized Picture Pool for Oral
Naming (PEDOI; Kremin et al., 2003). Our results also suggest that research involving pictorial processing or object naming across a wide age range should use norms specific to each age group to greatly reduce the impact of such confounding variables. Our results also suggest that researchers should be cautious when using norms from other cultures or other languages and when mixing norms from different databases.

\section{REFERENCES}

Alario, F. X., \& Ferrand, L. (1999). A set of 400 pictures standardized for French: Norms for name agreement, image agreement, familiarity, visual complexity, image variability, and age of acquisition. Behavior Research Methods, Instruments, \& Computers, 31, 531-552.

Bachoud-Lévi, A.-C., Dupoux, E., Cohen, L., \& Mehler, J. (1998). Where is the length effect? A cross-linguistic study of speech production. Journal of Memory \& Language, 39, 331-346.

Balota, D. A., \& Chumbley, J. I. (1990). Where are the effects of frequency in visual word recognition tasks? Right where we said they were! Comment on Monsell, Doyle, and Haggard (1989). Journal of Experimental Psychology: General, 119, 231-237.

Barry, C., Hirsch, K. W., Johnston, R. A., \& Williams, C. L. (2001). Age of acquisition, word frequency, and the locus of repetition priming of picture naming. Journal of Memory \& Language, 44, 350-375.

Barry, C., Morrison, C. M., \& Ellis, A. W. (1997). Naming the Snodgrass and Vanderwart pictures: Effects of age of acquisition, frequency and name agreement. Quarterly Journal of Experimental Psychology, 50A, 560-585.

Bates, E., Burani, C., D’Amico, S., \& Barca, L. (2001). Word reading and picture naming in Italian. Memory \& Cognition, 29, 986-999.

Baudot, J. (1992). Fréquences d'utilisation des mots en français écrit contemporain. Montréal: Presses de l'Université de Montréal.

Bonin, P., Chalard, M., Méot, A., \& Fayol, M. (2002). The determinants of spoken and written picture naming latencies. British Journal of Psychology, 93, 89-114.

Bonin, P., Peereman, R., Malardier, N., Méot, A., \& Chalard, M. (2003). A new set of 299 pictures for psycholinguistic studies: French norms for name agreement, image agreement, conceptual familiarity, visual complexity, image variability, age of acquisition, and naming latencies. Behavior Research Methods, Instruments, \& Computers, 35, 158-167.

Brown, A. S. (1991). A review of the tip-of-the-tongue experience. Psychological Bulletin, 109, 204-223.

Carroll, J. B., \& White, M. N. (1973). Word frequency and age of acquisition as determiners of picture-naming latency. Quarterly Journal of Experimental Psychology, 25, 85-95.

Chalard, M., Bonin, P., Méot, A., Boyer, B., \& Fayol, M. (2003). Objective age-of-acquisition (AoA) norms for a set of 230 object names in French: Relationships with psycholinguistic variables, the English data from Morrison et al. (1997), and naming latencies. European Journal of Cognitive Psychology, 15, 209-245.

Cuetos, F., Ellis, A. W., \& Alvarez, B. (1999). Naming times for the Snodgrass and Vanderwart pictures in Spanish. Behavior Research Methods, Instruments, \& Computers, 31, 650-658.

Dell' Acqua, R., LotTo, L., \& Job, R. (2000). Naming times and standardized norms for the Italian PD/DPSS set of 266 pictures: Direct comparisons with American, English, French, and Spanish published databases. Behavior Research Methods, Instruments, \& Computers, 32, 588-615.

Ellis, A. W., \& Morrison, C. M. (1998). Real age-of-acquisition effects in lexical retrieval. Journal of Experimental Psychology: Learning, Memory, \& Cognition, 24, 515-523.

Forster, K. I., \& CHAmbers, S. M. (1973). Lexical access and naming time. Journal of Verbal Learning \& Verbal Behavior, 12, 627-635.

Gilhooly, K. J., \& Gilhooly, M. L. (1979). Age-of-acquisition effects in lexical and episodic memory tasks. Memory \& Cognition, 7, 214-223.

Gilhooly, K. J., \& Gilhooly, M. L. (1980). The validity of age-ofacquisition ratings. British Journal of Psychology, 71, 105-110. 
Gilhooly, K. J., \& Logie, R. H. (1980). Age-of-acquisition, imagery, concreteness, familiarity, and ambiguity measures for 1,944 words. Behavior Research Methods \& Instrumentation, 12, 395-427.

Hodgson, C., \& Ellis, A. W. (1998). Last in, first to go: Age of acquisition and naming in the elderly. Brain \& Language, 64, 146-163.

Huttenlocher, J., \& Kubicek, L. F. (1983). The source of relatedness effects on naming latency. Journal of Experimental Psychology: Learning, Memory, \& Cognition, 9, 486-496.

JescheniaK, J. D., \& LeVelt, W. J. M. (1994). Word frequency effects in speech production: Retrieval of syntactic information and of phonological form. Journal of Experimental Psychology: Learning, Memory, \& Cognition, 20, 824-843.

Kremin, H., Akhutina, T., Basso, A., Davidoff, J., De Wilde, M., KitzING, P., ET AL. (2003). A cross-linguistic data bank for oral picture naming in Dutch, English, German, French, Italian, Russian, Spanish, and Swedish (PEDOI). Brain \& Cognition, 53, 243-246.

Kremin, H., Hamerel, M., Dordain, M., De Wilde, M., \& Perrier, D. (2000). Age of acquisition and name agreement as predictors of mean response latencies in picture naming of French adults. Brain \& Cognition, 43, 286-291.

Kremin, H., Perrier, D., De Wilde, M., Dordain, M., Le Bayon, A., GatignOL, P., ET AL. (2001). Factors predicting success in picture naming in Alzheimer's disease and primary progressive aphasia. Brain \& Cognition, 46, 180-183.

Lachman, R., Shaffer, J. P., \& Hennrikus, D. (1974). Language and cognition: Effects of stimulus codability, name-word frequency, and age of acquisition on lexical reaction time. Journal of Verbal Learning \& Verbal Behavior, 13, 613-625.

Meschyan, G., \& Hernandez, A. (2002). Age of acquisition and word frequency: Determinants of object-naming speed and accuracy. Memory \& Cognition, 30, 262-269.

Monsell, S. (1991). The nature and locus of word frequency effects in reading. In D. Besner \& G. W. Humphreys (Eds.), Basic processes in reading: Visual word recognition (pp. 148-197). Hillsdale, NJ: Erlbaum.

Morrison, C. M., Chappell, T. D., \& Ellis, A. W. (1997). Age of acquisition norms for a large set of object names and their relation to adult estimates and other variables. Quarterly Journal of Experimental Psychology, 50A, 528-559.

Morrison, C. M., Ellis, A. W., \& Quinlan, P. T. (1992). Age of acquisition, not word frequency, affects object naming, not object recognition. Memory \& Cognition, 20, 705-714.

New, B., Pallier, C., Ferrand, L., \& Matos, R. (2001). Une base de données lexicales du français contemporain sur internet: LEXIQUE. L'Année Psychologique, 101, 447-462.

OldField, R. C., \& Wingfield, A. (1965). Response latencies in naming objects. Quarterly Journal of Experimental Psychology, 17, 273 281.

Paivio, A., Clark, J. M., Digdon, N., \& Bons, T. (1989). Referential processing: Reciprocity and correlates of naming and imaging. Memory \& Cognition, 17, 163-174.

PAquot, A. (1988). Les Québécois et leurs mots: Étude sémiologique et sociolinguistique des régionalismes lexicaux au Québec. Sainte-Foy: Presses de l'Université Laval.

Sanfeliu, M. C., \& Fernandez, A. (1996). A set of 254 SnodgrassVanderwart pictures standardized for Spanish: Norms for name agreement, image agreement, familiarity, and visual complexity. Behavior Research Methods, Instruments, \& Computers, 28, 537-555.

SnOdgrass, J. G., \& VANDERWART, M. (1980). A standardized set of 260 pictures: Norms for name agreement, image agreement, familiarity, and visual complexity. Journal of Experimental Psychology: Human Learning \& Memory, 6, 174-215.

SnOdgrass, J. G., \& Yuditsky, T. (1996). Naming times for the Snodgrass and Vanderwart pictures. Behavior Research Methods, Instruments, \& Computers, 28, 516-536.

Statistics CANADA (1996). Total, average, and median years of schooling, age groups and sex for population 15 years and over, for Canada,

provinces, territories, census metropolitan areas and census agglomerations, 1991 to 2001 censuses. www.statcan.ca/losolc?catno=97F0 $017 \mathrm{X} 2001008$.

Vitkovitch, M., \& Tyrrell, L. (1995). Sources of disagreement in object naming. Quarterly Journal of Experimental Psychology, 48A, 822-848.

APPENDIX

\section{Items That Did Not Reach an \\ Overall Name Agreement of 33\%}

\begin{tabular}{ll}
\hline \multicolumn{1}{c}{ English } & \multicolumn{1}{c}{ French } \\
\hline barracks & caserne \\
blueberries & bleuets \\
bricklayer & briqueleur \\
cash register & caisse \\
chestnut & châtaignes \\
corn & blé d'Inde \\
cross & croix \\
deer & chevreuil \\
eagle & aigle \\
fields & champs \\
flower & fleur \\
gallows & potence \\
glue & colle \\
key & clé \\
rock & rocher \\
shore & rivage \\
shorts & short \\
slingshot & sling shot \\
sneaker & espadrilles \\
suit & tailleur \\
trombone & trombone \\
tweezers & pince à sourcils \\
umbrella stand & porte-parapluie \\
uniform & uniforme \\
veil & voile \\
vines & vignes \\
\hline & \\
&
\end{tabular}

Items That Were Removed Because Their Name Is Polysemous

\begin{tabular}{ll}
\hline \multicolumn{1}{c}{ English } & French \\
\hline binoculars & jumelles \\
cards & cartes \\
flower & fleur \\
flower & fleur \\
flower & fleur \\
flowers & fleurs \\
office & bureau \\
office & bureau \\
office & bureau \\
peach & pêche \\
secretary & secrétaire \\
tower & tour \\
\hline
\end{tabular}

(Manuscript received September 24, 2004; revision accepted for publication May 20, 2005.) 